\title{
RESISTÊNCIA À FLEXÃO DE COMPÓSITOS DE MATRIZ EPÓXI REFORÇADOS COM TECIDO DE RAMI SOB CONDIÇÕES DE VÁCUO*
}

\author{
Janine Feitosa de Deus ${ }^{1}$ \\ Caroline Gomes de Oliveira ${ }^{2}$ \\ Lucas de Almeida Pontes ${ }^{3}$ \\ Rubén Jesus Sanchez Rodriguez ${ }^{4}$ \\ Frederico Muylaert Margem ${ }^{5}$ \\ Sérgio Neves Monteiro ${ }^{6}$ \\ Felipe Perissé Duarte Lopes
}

Resumo

Compósitos ambientalmente corretos, fabricados a partir de fibras naturais lignocelulósicas (FNL), estão entre os mais investigados e aplicados atualmente. Dentre as FNL, destacam-se as fibras de Rami (Boehmeria nivea), que surgem como alternativa em sistemas de blindagens multicamadas, para uso em coletes de proteção balística, por serem leves, de baixo custo, resistentes e ambientalmente corretos. No presente trabalho, investigou-se o comportamento mecânico de compósitos de matriz epóxi reforçados com tecido de rami, através de testes de flexão. Compósitos contendo separadamente $0,10,20$ e 30\% em volume de tecido de rami e resina epóxi, foram fabricados em um molde metálico, sob condições de vácuo. Os corpos de prova fraturados foram analisados em MEV e os resultados foram avaliados visando algumas propriedades de flexão com a incorporação do tecido de rami, visando sua posterior aplicação em blindagem balística.

Palavras-chave: Compósitos; Tecido de Rami; Fibras Naturais Lignocelulósicas; Resina Epóxi.

\section{BENDING RESISTANCE OF EPOXY COMPOSITES REINFORCED WITH RAMI FABRIC UNDER VACUUM CONDITIONS}
Abstract
Environmentally correct composites, made from natural lignocellulosic fibers (FNL), are among the most researched and applied today. Among the FNLs, we highlight the Rami fibers (Boehmeria nivea), which arise as fabrics in multilayer armor systems, for use in ballistic protection vests, because they are lightweight, low cost, good resistance and environmentally correct. In the present work, the mechanical behavior of epoxy matrix composites reinforced with ramie fabric was investigated through bending tests. Composites containing 0, 10, 20 and 30 vol\% of ramie fabric and epoxy resin were fabricated in a metal mold under vacuum conditions. The fractured specimens were analyzed in SEM and the results were evaluated aiming at some flexural properties with the incorporation of ramie fabric, aiming at their subsequent application in ballistic armor.
Keywords: Composites; Rami fabric; Natural Lignocellulosic Fibers; Epoxy.
1 Doutorando, Laboratório de Materiais Avançados - LAMAV, Centro de Ciência e Tecnologia, Universidade Estadual do Norte Fluminense, CCT/UENF, Av. Alberto Lamego, 2000, 28015-620, Campos dos Goytacazes, RJ-Brasil.
2 Engenheira Metalúrgica e de Materiais, Laboratório de Materiais Avançados - LAMAV, Centro de Ciência e Tecnologia, Universidade Estadual do Norte Fluminense, CCT/UENF. Campos dos Goytacazes, RJ - Brasil. Graduando em Engenheira Metalúrgica e de Materiais, LAMAV - CCT/UENF.
PhD, Professor Titular, LAMAV - CCT/UENF.
Doutor em Engenharia Metalúrgica e de Materiais, Professor, Faculdade Redentor - Itaperuna, RJ - Brasil.
PhD, Professor Titular, Instituto Militar de Engenharia - IME. Rio de Janeiro, RJ - Brasil
Doutor em Engenharia Metalúrgica e de Materiais, LAMAV - CCT/UENF. 


\section{INTRODUÇÃO}

Ao decorrer dos anos o desenvolvimento mundial vem necessitando de materiais que apresentem custos baixos e que não prejudiquem o meio ambiente. Conflitos relacionados com a utilização de formas não renováveis de energia, estão aumentando o interesse de introduzir ao mercado materiais naturais em substituição aos sintéticos, pois os materiais sintéticos apresentam um maior consumo de energia em sua fabricação.

Tecidos de fibras sintéticas de alto desempenho, como a aramida (Kevlar® e Twaron $\AA$ ) e o polietileno de ultra alto peso molecular (Spectra $\AA^{\circledR}$ e Dyneema $\left.{ }^{\circledR}\right)$, têm sido utilizados na segunda camada da blindagem [1]. No entanto estas fibras são caras, não-renováveis e tem vida útil relativamente curta. Neste cenário, os compósitos reforçados com fibras naturais lignocelulósicas (FNL) surgem como alternativa aos tecidos de fibras sintéticas em sistemas de blindagens multicamadas, para uso em coletes de proteção balística, por serem leves, de baixo custo e ambientalmente corretos [2]. Estes materiais podem apresentar boa eficiência de proteção balística, e nos últimos anos, há um crescente interesse dos pesquisadores em desenvolver as propriedades destes compósitos para a presente aplicação [3-7]. Tendo em vista as boas propriedades das FNL, bem como o potencial brasileiro para sua produção, é de grande interesse a pesquisa de novas aplicações para estas fibras, especialmente aplicações de alta tecnologia, em produtos de mais alto valor agregado que beneficiem as comunidades produtoras.

O rami (Boehmeria nivea), conhecido comercialmente como grama da China, é originário do sudeste asiático e produz fibras que podem ser extraídas do floema da planta. É comumente utilizada como forrageira pela pecuária e empregada pela indústria têxtil na fabricação de tecidos utilizados em diversos fins, como roupas, decorações de interiores e até mesmo velame de paraquedas [8-9]. Apesar de apresentar as vantagens comuns das fibras naturais lignocelulósicas, seu uso como reforço em materiais compósitos ainda não é difundido.

Assim, o presente trabalho tem como objetivo avaliar o comportamento mecânico de compósitos de matriz epóxi reforçados com tecido de rami, fabricados sob condições de vácuo, quando submetidos à esforços de flexão. Para tal foram realizados ensaios de flexão em compósitos reforçados com 10, 20 e $30 \%$ em fração volumétrica de tecido de rami incorporado, visando sua posterior aplicação em blindagem balística.

\section{MATERIAIS E MÉTODOS}

A resina utilizada foi a epóxi, comercializada pela empresa Resinpoxy, localizada na cidade do Rio de Janeiro - RJ. Esta resina é do tipo éter diglicidílico do bisfenol A (DGEBA), endurecida com trietileno tetramina (TETA). O endurecedor foi adicionado à resina na proporção de $13 \%$ em peso, pois esta é a proporção estequiométrica que equivale à abertura de todos os anéis epóxi por ligação com as moléculas de amina. O tecido de rami utilizado neste trabalho foi importado da china da empresa Rose Natural Healthy Items Wholesale, a qual comprou o tecido de rami de um fabricante localizado na cidade chinesa de Guangzhou (Fig. 1). 


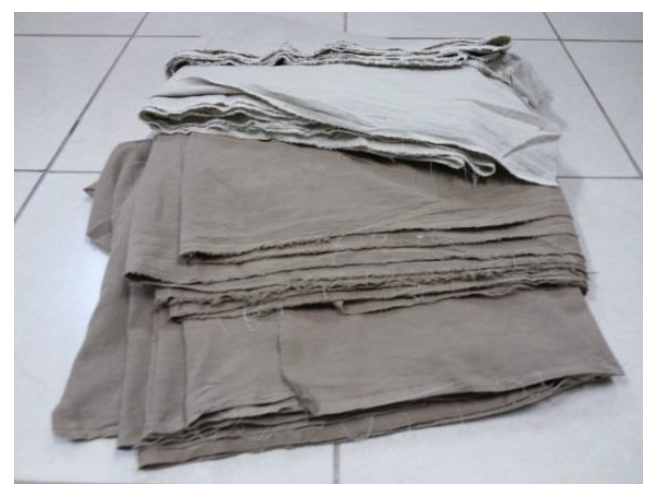

Figura 1 - Tecido de Rami

O tecido de rami foi seco em estufa a $60^{\circ} \mathrm{C}$ por $24 \mathrm{~h}$ para retirada da umidade, antes de sua utilização.

Os compósitos laminados foram formulados, manualmente, alternando-se as camadas de resina epóxi (já misturada com o endurecedor) e tecido de rami, em um molde metálico, mantido a vácuo através de uma bomba de sucção, garantindo a eliminação das bolhas e vazios no interior do compósito laminado. Após a fabricação, os compósitos laminados foram curados em temperatura ambiente por 24 horas e depois cortados em corpos de prova nas dimensões de $122 \times 25 \times 7 \mathrm{~mm}$ de acordo com a norma ASTM D 790 [10], para o ensaio de flexão. Os ensaios mecânicos de flexão foram realizados na máquina universal de ensaios mecânicos, marca Instron, modelo 5582, disponível no SMMA/LAMAV. Os valores de tensão de flexão $(\sigma)$, módulo de flexão $(E)$ e deformação $(\varepsilon)$ serão determinados pelas seguintes Equações:

$$
\begin{aligned}
& \sigma=3 P L / 2 b d^{2} \\
& \varepsilon=6 D d / L^{2} \\
& E=L^{3} m / 4 b d^{2}
\end{aligned}
$$

Onde: $\mathrm{P}$ é a carga, $\mathrm{b}$ e $\mathrm{d}$ a largura e a espessura da amostra respectivamente, $\mathrm{L}$ é 0 comprimento entre apoios, $\mathrm{D}$ é a deformação máxima do centro do feixe e $\mathrm{m}$ é o declive da parcela inicial em linha reta da curva de carga de deformação.

A superfície de fratura dos corpos de prova foi caracterizada, após cobertura com ouro, por microscopia eletrônica de varredura, MEV, modelo Shimadzu SSX-550, do laboratório de materiais avançados - LAMAV.

\section{RESULTADOS E DISCUSSÃO}

A Fig. 2 ilustra o aspecto típico das curvas de força vs. deformação, obtidas nos ensaios de flexão de três pontos, para corpos de prova representativos das diferentes frações de tecidos de juta. Nesta figura observa-se que, como esperado, a curva de flexão da resina epóxi pura é linear, correspondendo ao regime elástico até a ruptura, o que caracteriza um comportamento frágil. O mesmo acontece com a maioria das curvas dos compósitos com 10 e 20 vol\% de tecidos de juta, como exemplificado na Fig. 2, que também apresentam características frágeis. Isto significa que, ao se atingir o ponto de máxima resistência, a ruptura é súbita com imediata queda de praticamente toda a carga aplicada. 


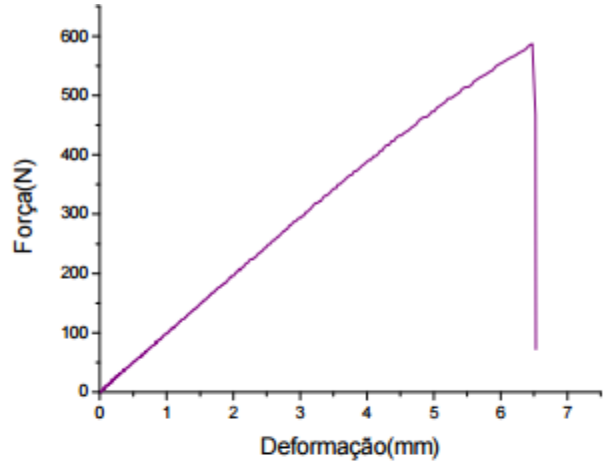

$0 \%$

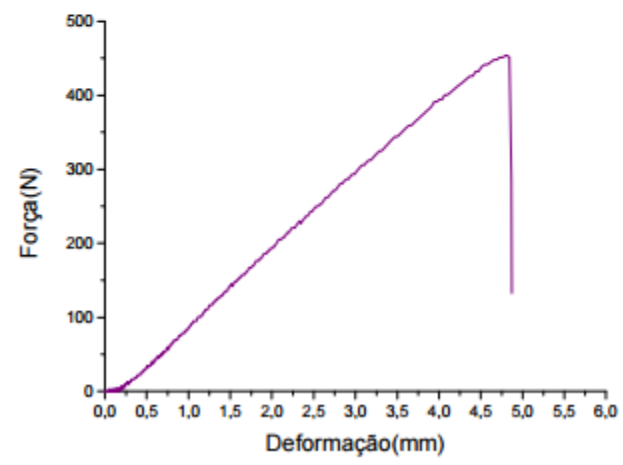

$20 \%$

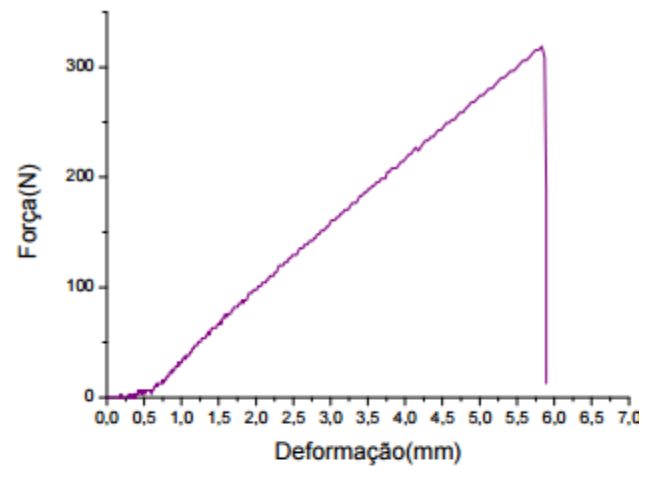

$10 \%$

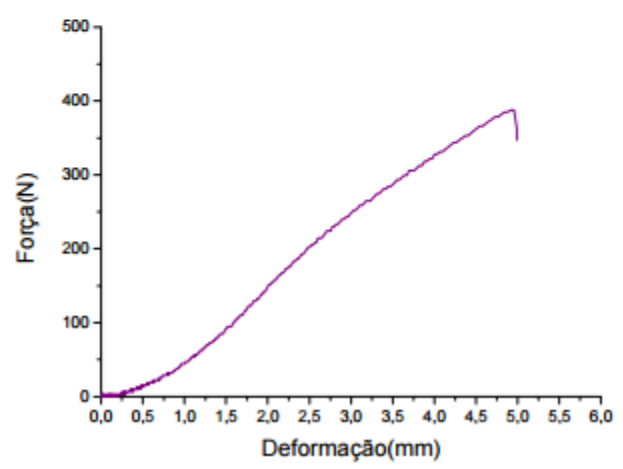

$30 \%$

Figura 2 - Curvas de carga vs. deslocamento dos ensaios de flexão de compósitos de matriz epóxi reforçados com diferentes frações de tecido de rami.

Nos compósitos com 30 vol\% de tecido, como o exemplo da Fig. 2, após um início linear, as curvas apresentam um trecho parabólico com suave inclinação característica de uma certa plasticidade. Além disto, ao se atingir o máximo, não ocorre uma fratura súbita e a carga oscila no seu lento decréscimo. Isto se deve à efetiva resistência que o maior número de fibras do tecido oferecem à propagação de trincas, através da frágil matriz de epóxi. Ou seja, com 30 vol\% de tecido, não existirá mais um percurso fácil através das fibras do tecido, que permita uma única trinca propagar-se imediatamente por toda a seção do corpo de prova para súbita ruptura, como acontece com os compósitos com 10 e 20 vol\% de fibra na Fig. 2. Em conseqüência, a total ruptura dos corpos de prova com 30 vol\% de tecido de juta só ocorre após extensa deflexão plástica dos mesmos. Isto está associado a uma elevada tenacidade destes compósitos.

A partir de curvas como as da Fig. 2, obteve-se o valor das forças máximas, Fm, e as deformações correspondentes, $\varepsilon$, e calculou-se, pelas Eq. [1] e [3], a tensão máxima, om, e o módulo de elasticidade, Em. A Tabela 1 apresenta as médias da resistência à flexão e módulo de elasticidade, resultantes de compósitos de epóxi com diferentes frações de volumes de tecido de rami. 
Tabela 1 - Resistência à flexão e modulo de elasticidade para compósitos de epóxi reforçados com tecido de rami.

\begin{tabular}{c|cc}
\hline $\begin{array}{c}\text { Fração volumétrica de } \\
\text { fibra de juta (\%) }\end{array}$ & $\begin{array}{c}\text { Resistência à } \\
\text { flexão }(\mathbf{M P a})\end{array}$ & $\begin{array}{c}\text { Módulo de } \\
\text { Elasticidade (GPa) }\end{array}$ \\
\hline 0 & $62.0 \pm 6.0$ & $2.28 \pm 0.25$ \\
10 & $53,1 \pm 10.7$ & $2,47 \pm 0.16$ \\
20 & $34,4 \pm 16.3$ & $1,06 \pm 0.66$ \\
30 & $10,6 \pm 7.3$ & $0,20 \pm 0.24$ \\
\hline
\end{tabular}

Com base nestes valores, construíram-se os gráficos de variação da resistência à flexão e módulo de elasticidade dos compósitos em função da fração em volume de tecido de rami, como mostrado na Figura 3.
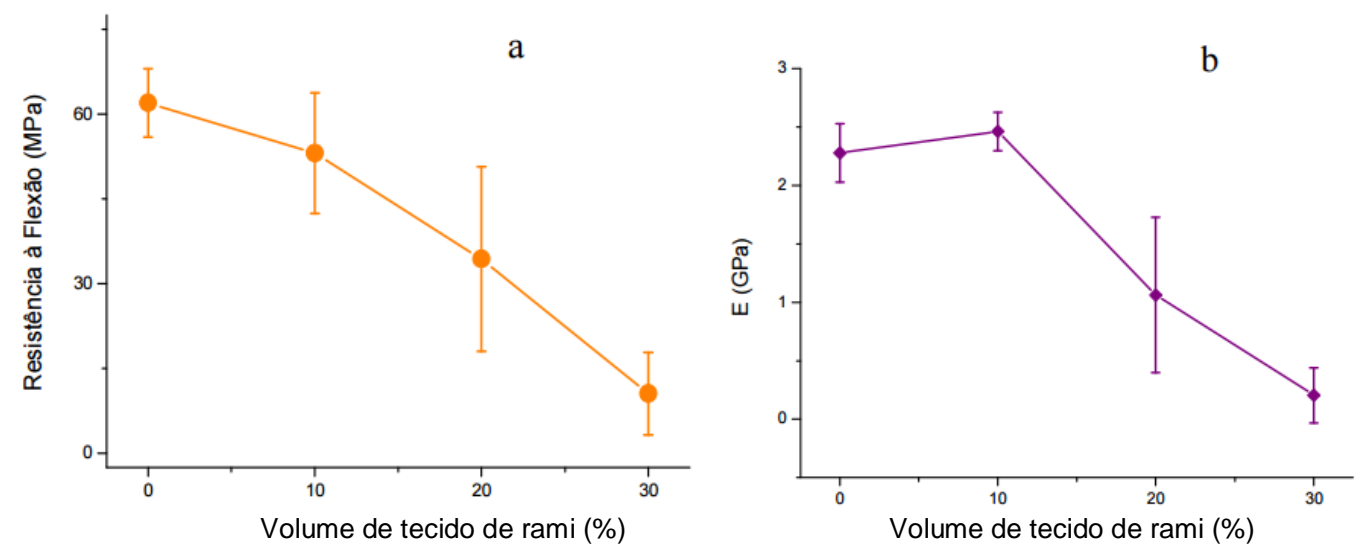

Figura 3 - Variação da resistência à flexão dos compósitos de matriz epóxi com quantidade incorporada de tecido de rami.

No gráfico da Fig. 3 (a) nota-se um decréscimo na resistência dos compósitos em relação à resistência da resina epóxi pura, 0 vol\% de tecido. Com a incorporação do tecido de rami, a matriz epóxi atinge, em média, resistências inferiores a 60 MPa. É importante mencionar que os valores obtidos podem estar relacionados a uma baixa adesão do tecido à resina, o que ocasiona, em princípio, uma dúvida quanto à eficácia do reforço do tecido de rami na matriz epóxi. Isto sugere que uma investigação, com um número de corpos de prova bem maior do que os seis (para cada fração) do presente trabalho, deve ser realizada. Esta interpretação dos resultados dos ensaios de flexão na Fig. 3 indicaria que o tecido de rami não se constituem em efetivo reforço para compósitos com matriz epóxi. Isto é, à medida que se incorpora tecido de rami à matriz ocorre um decréscimo marcante na resistência, o que pode ser atribuído aos vazios decorrentes do processamento manual que dificulta a impregnação do tecido pela resina.

O módulo de elasticidade (Fig. 3 b), aumenta para o compósito de uma fração de volume de 10 vol\% e depois diminui para as demais frações volumétricas. Esse comportamento do módulo de elasticidade ocorre, pois as frações de volume de fibra acima de 20 vol\% são difíceis de serem processadas para garantir uma estrutura composta sem poros e falhas [11-12]. O comportamento na fratura dos compósitos investigados revelou alguns detalhes importantes. A Fig. 4 mostra o aspecto visual dos compósitos que foram rompidos em ensaios de flexão. Nesta figura, os principais aspectos a serem observados são a ruptura frágil dos corpos de prova de 
0 e 10 vol\% de tecido, bem como a aparente não-ruptura das espécies de 20 e 30 vol\% de tecido [13]. Na verdade, até 10 vol\% de tecido, a fratura ocorre transversalmente na matriz de epóxi e como o tecido de rami é relativamente pouco, não oferecem um obstáculo à propagação de trincas através da matriz. Em contrapartida, para frações volumétricas acima de 20 e $30 \%$ de tecido (Fig. 4), a fratura ocorre principalmente por delaminação entre as fibras do tecido e a matriz.

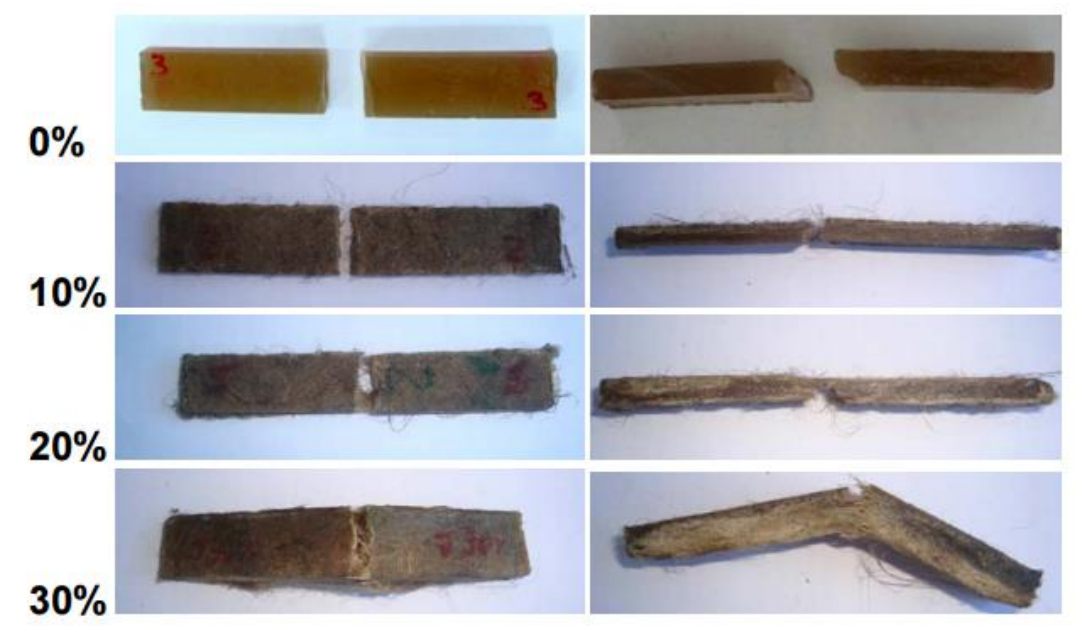

Figura 4 - Aspecto visual dos corpos de prova rompidos: (a) visão frontal; (b) visão lateral.

A Fig. 5 mostra micrografias típicas da ruptura de compósitos de 30 vol\% de tecido de rami em matriz epóxi, obtidas em MEV. Nesta figura, pode-se notar, com menor ampliação (Fig. 5 a), a matriz de epóxi rompida com fibras de tecido incorporadas. Com maior aumento (Fig. 5 b), pode-se ver com evidência a descolagem fibras de tecido / matriz. Esta parece ser uma conseqüência da tensão interfacial de cisalhamento relativamente baixa associadas à interação das fibras de tecido de rami com uma matriz polimérica.
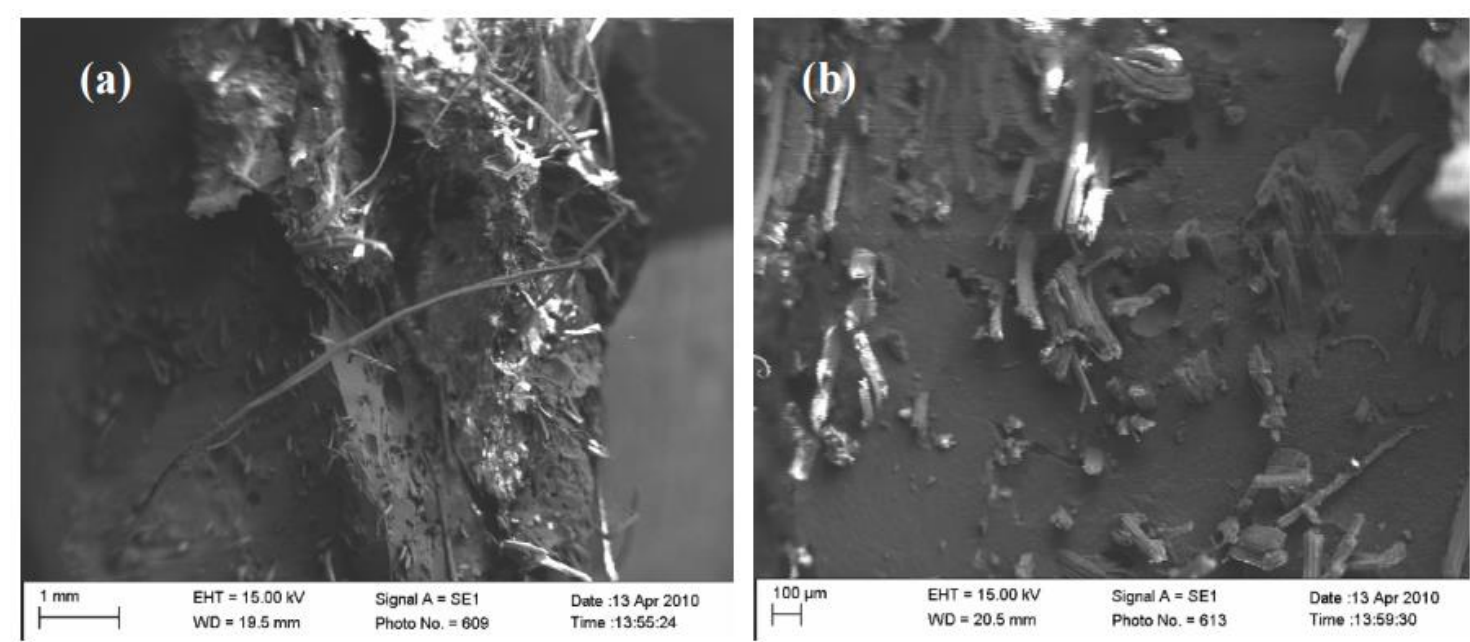

Figura 5 - Micrografia obtida em MEV de compósitos de 30\% de tecido de rami reforçados com resina epóxi: (a) 30X e (b) 100X. 


\section{CONCLUSÃO}

- Compósitos de matriz epóxi incorporada com tecido de rami apresentam um decréscimo na resistência à medida que se aumenta a fração volumétrica de tecido.

- O módulo de elasticidade passa por um máximo para $10 \%$ em volume de tecido de rami e depois também decresce. Incorporações de tecido de rami reduzem a resistência do compósito com matriz epóxi possivelmente devido a dificuldades de processamento manual que não garante boa impregnação dos tecidos pela resina epóxi.

- O tecido de rami, embora tenham elevada resistência mecânica, está associado a uma fraca interface com a matriz de epóxi. Isto permite que trincas que esbarram nas fibras do tecido prossigam através da sua superfície, contribuindo para a ruptura do compósito.

\section{AGRADECIMENTOS}

Os autores agradecem ao suporte financeiro e de bolsas concedidos pelo CNPq, CAPES e FAPERJ.

\section{REFERÊNCIAS}

[1] Mobasseri AA, Ansari AR, Zarei HR, Sedighi M, Mobasseri F. Optimization of Combined Layers Produced by the Ceramic/Composite and Ceramic/Aluminum Plates. Australian Journal of Basic and Applied Sciences, Vol. 7, n.6, p. 199-210. 2013.

[2] Faruk P, Bledzki AK, Fink H, Sain M. Progress Report on Natural Fiber Reinforced Composites. Macromolecular Materials Engineering, Vol. 299, n.1, p. 9-26. 2014.

[3] Abidin MHZ, Mohamad MAH, Zaidi AMA, Mat, W.A.W. Experimental Study on Ballistic Resistance of Sandwich Panel Protection Structure with Kenaf Foam as a Core Material against Small Arm Bullet. Applied Mechanics and Materials, Vol. 315, p. 612-615. 2013.

[4] Marsyahyo E, Jamasri, Rochardjo HSB, Soekrisno. Preliminary Investigation on Bulletproof Panels Made from Ramie Fiber Reinforced Composites for NIJ Level II, IIA, and IV. Journal of Industrial Textiles, Vol. 39, p. 13-26, 2009.

[5] Monteiro SN, Louro LHL, Trindade W, Elias CN, Ferreira CL, Lima ES, et al. Natural Curaua Fiber-Reinforced Composites in Multilayered Ballistic Armor. Metallurgical and Materials Transactions A (On-line). Disponível em: <http://link.springer.com /article/10. 1007\%2Fs11661-015-3032-z>. 2015.

[6] Radif ZS, Ali A, Abdan K. Development of a Green Combat Armour from Rame-Kevlar-Polyester Composite. Pertanika Journal of Science and Technology, Vol. 19, nํ2, p. 339-348. 2011.

[7] Wambua P, Vangrimde B, Lomov S, Verpoest I. The Response of Natural Fibre Composites to Ballistic Impact by Fragment Simulating Projectiles. Composite Structures, Vol. 77, p. 232-240. 2007.

[8] Freitas CFT, Aspectos econômicos da cultura de rami. Instituto de Economia Agrícola (IEA), São Paulo.

[9] Swicofil. Natural Fibers: Ramie. 2015. Disponível em: http://www.swicofil.com/products/007ramie.html, acessado em 10/04/2016. 
[10] ASTM - American Society for Testing Materials. ASTM D790 - 03 - Standard Test Methods for Flexural Properties of Unreinforced or Reinforced Plastics and Electrical Insulating Materials. Annual Book of ASTM, 2003.

[11] Monteiro SN, Aquino RCMP, Lopes FPD, Carvalho EA, D'Almeida JRM. Comportamento mecânico e características estruturais de compósitos poliméricos reforçados com fibras contínuas e alinhadas de curauá. Revista Matéria, v. 11, n. 3, p. 197-203, 2006.

[12] Agarwal BD, Brountman LJ. Analysis and Performance of Fiber Composites, Nova York: John Wiley \& Sons, 1990. 13 Ashbee, K.H.G. Fundamental Principles of Fiber Reinforced Composites, Lancaster, Inglaterra: Technomic Pub. Co. Inc., 1993.

[13] Aquino RCMP, D'Almeida JRM, Monteiro SN. Flexural Mechanical Porperties of Piassava Fibers (Attalea funifera)-Resin Matrix Composites. Journal of Materials Science Letters, v. 20, pp. 1017-1019, 2001. 